\title{
Estimativa da ÁREA FoliAR DE Euphorbia heterophylla
}

\author{
Euphorbia heterophylla Leaf Area Estimate
}

\begin{abstract}
DUARTE, D.J. ${ }^{2}$, BIANCO, S. ${ }^{3}$, LEONARDO B.C. ${ }^{4}$ e PANOSSO, A.R. ${ }^{4}$
RESUMO - A estimativa da área foliar pode auxiliar na compreensão de relações de interferência entre plantas daninhas e cultivadas. Com o objetivo de obter uma equação que, através de parâmetros lineares dimensionais das folhas, permita determinar a área foliar estimada (Af') de Euphorbia heterophylla, estudaram-se relações entre a área foliar real e os parâmetros dimensionais do limbo foliar, como o comprimento ao longo da nervura principal (C) e a largura máxima (L) perpendicular à nervura principal. Foram analisados 200 limbos foliares, coletados em diferentes agroecossistemas na Universidade Estadual Paulista, campus de Jaboticabal. Os modelos estatísticos utilizados foram: linear, $\mathrm{Y}=\mathrm{a}+$ bx; linear simples, $\mathrm{Y}=\mathrm{bx}$; geométrico, $\mathrm{Y}=\mathrm{ax}^{\mathrm{b}}$; e exponencial, $\mathrm{Y}=\mathrm{ab}^{\mathrm{x}}$. Todos os modelos analisados podem ser empregados na estimação da área foliar de E. heterophylla. Sugere-se optar pela equação linear simples envolvendo $C * L$, considerando o coeficiente linear igual a zero, em razão de sua praticidade. Desse modo, a estimativa da área foliar de $E$. heterophylla pode ser obtida pela equação $A f^{\prime}=0,6816^{*}\left(C^{*} L\right)$.
\end{abstract}

Palavras-chave: amendoim-bravo, limbo foliar, planta daninha.

\begin{abstract}
Leaf area estimate may contribute to understand the relationships of interference among weeds and crops. The objective of this research was to obtain a mathematical equation to estimate the leaf area of Euphorbia heterophylla based on linear measures of the leaf blade. Correlation studies were carried out using the real leaf area and leaflength (C) and the maximum leaf width (L) of 200 leaf blades which were collected from several agroecosystems at Universidade Estadual Paulista in Jaboticabal, SP, Brazil. The evaluated statistic models were: linear $Y=a+$ $b x$; simple linear $Y=b x$; geometric $Y=a x^{b}$; and exponential $Y=a b^{x}$. All of the evaluated models can be used for $\boldsymbol{E}$. heterophylla leaf area estimation. The simple linear regression model is suggested using $C^{*} L$ and taking the linear coefficient equal to zero. Thus, an estimate of the leaf area of E. heterophylla can be obtained using the equation $A f^{\prime}=0.6816^{*}\left(C^{*} L\right)$.
\end{abstract}

Keywords: wild poinsettia, leaf blade, weed

\section{INTRODUÇÃO}

Euphorbia heterophylla, cujo centro de origem compreende Brasil e Paraguai, está amplamente distribuída na região Centro-Sul do Brasil, infestando diversas culturas de interesse econômico. É uma planta de ciclo anual com porte médio $(40-60 \mathrm{~cm})$, rápido crescimento e multiplicação por sementes, sendo altamente competitiva com as culturas agrícolas; o caule é simples ou ramificado, com modificações em intervalos regulares; as folhas são alternas, opostas ou verticiladas, ocorrendo tanto no caule como nos ramos, com maior concentração na parte final destes, abaixo da inflorescência (Kissmann \& Groth, 1999).

Recebido para publicação em 9.6.2008 e na forma revisada em 21.8.2009.

2 Mestrando do Programa de Pós-Graduação em Produção Vegetal da FCAV/UNESP, Jaboticabal-SP, <duarte_dj@yahoo.com.br>;

${ }^{3}$ Professor Livre-Docente do Dep. de Biologia Aplicada à Agropecuária, Faculdade de Ciências Agrárias e Veterinárias, Universidade Estadual Paulista, FCAV/UNESP, Via de Acesso Prof. Paulo Donato Castellane s/ n, 14884-900 Jaboticabal-SP; ${ }^{4}$ Doutorando do Programa de Pós-Graduação em Produção Vegetal da FCAV/UNESP, Jaboticabal-SP. 
Estudos básicos a respeito de reprodução, crescimento e desenvolvimento, exigência nutricional, respostas aos sistemas de controle e outros aspectos são necessários para melhor compreensão da biologia e escolha de métodos de manejo de espécies daninhas. Na maioria desses estudos, o conhecimento da área foliar é fundamental, pois é talvez o mais importante parâmetro na avaliação do crescimento vegetal, além de auxiliar na compreensão de relações de interferência entre plantas daninhas e cultivadas (Bianco et al., 2008a, b).

A estimativa da área foliar por meio de equações matemáticas tem sido utilizada com boa precisão, sendo um método fácil, rápido e não destrutivo, tornando-se importante para avaliar o crescimento das plantas nas condições de campo (Bianco et al., 2007a,b). Equações de regressão, relacionando área foliar real com parâmetros dimensionais lineares das folhas, para estimativa de área foliar têm sido utilizadas com sucesso tanto em plantas cultivadas (Pedro Júnior et al., 1986; Silva et al., 1998; Queiroga et al., 2003) quanto em plantas daninhas (Bianco et al., 2002, 2005, 2007a, b).

O objetivo deste trabalho foi determinar uma equação matemática adequada para estimar a área foliar de E. heterophylla, a partir de dimensões lineares dos limbos foliares.

\section{MATERIAL E MÉTODOS}

Esta pesquisa foi realizada na Faculdade de Ciências Agrárias e Veterinárias da Universidade Estadual Paulista (FCAV/ UNESP), campus de Jaboticabal. As folhas utilizadas no estudo foram coletadas em meados do mês de maio de 2008, em diferentes agroecossistemas da FCAV/UNESP, buscando amostrar plantas de $E$. heterophylla nas diferentes condições ecológicas em que a espécie pode ocorrer dentro de ambientes agrícolas. Foram coletados 200 limbos foliares, considerando todas as folhas das plantas, desde que não apresentassem deformações oriundas de fatores externos, como pragas, moléstias e granizo.

Em cada local de amostragem, 10 a 20 folhas de diferentes plantas foram coletadas e, em seguida, rapidamente levadas ao Laboratório de Biologia e Manejo de Plantas Daninhas da FCAV/UNESP, para determinação do comprimento do limbo foliar ao longo da nervura principal $(C)$ e da largura máxima do limbo foliar $(L)$ perpendicular à nervura principal. A seguir, suas áreas foliares reais $(A f)$ foram determinadas, com a utilização do aparelho eletrônico Portable Area Meter Licor mod. L1 - 3000.

Para escolha de uma equação que possa representar a área foliar estimada $\left(A f^{\prime}\right)$ em função das dimensões do limbo foliar, procedeu-se aos estudos de regressão utilizando as seguintes equações: linear, $Y=a+b x$; linear pela origem, $Y=b x$; geométrica, $Y=a x^{b}$; e exponencial, $Y=a b^{x}$, em que o valor $Y$ estima a área do limbo foliar em função de $X$, cujos valores podem ser $C, L$ ou $C^{*} L$. No caso de $x$ igual a $C^{*} L$, estimou-se também a equação linear passando pela origem, o que significa supor que a área é proporcional a um retângulo $C^{*} L$.

Todas as equações utilizadas são lineares ou linearizáveis por transformação, de modo que os ajustes foram feitos a partir de retas. Para realizar as comparações entre os modelos, foram obtidas as somas de quadrados das diferenças entre os valores observados e os preditos pelos modelos, denominando isso de soma de quadrados do resíduo. No caso dos modelos com transformação (geométrica e exponencial), foi feita a volta para a escala original e, após isso, obtida as referidas somas de quadrados do resíduo.

Os coeficientes de correlação são os obtidos com as variáveis de trabalho $x$ e $Y$, no caso linear; logaritmo de $Y$ e logaritmo de $x$, no caso geométrico; e logaritmo de $Y$ e $x$, no caso exponencial. $\mathrm{O}$ número de graus de liberdade (GL) é o número de folhas analisadas menos o número de parâmetros estimados para cada mode1o. Para testar o acréscimo de soma de quadrados do resíduo (SDRes) do modelo passando pela origem, em relação ao modelo com intercepto, utilizou-se o teste $\mathrm{F}$ condicional: $\mathrm{F}=(\mathrm{SQRes}$ $(0,0)$ - SQRes CL) / SQRes CL/GL), com 1 e $2 \mathrm{GL}$, sendo GL o número de folhas menos 2 (Neter \& Wasserman, 1974; Mead \& Curnow, 1983), em que SQRes $(0,0)$ é igual à SQRes do modelo linear passando pela origem (modelo $Y$ $=b x)$ e a SQRes $(C L)$ é igual à SQRes do modelo linear com parâmetros $a$ e $b(Y=a+b x)$. A equação de regressão com menor SQRes na escala real (sem transformação) é a que melhor estima a área foliar (Peressin et al., 1984). 
Para verificar a aceitação dos modelos matemáticos obtidos, foram efetuadas análises estatísticas, testando a relação entre os valores reais de área foliar e os valores estimados pela equação escolhida. Para isso, foram aplicados os testes de normalidade de ShapiroWilk e de correlação de Spearman, a 5\% de significância. Essa verificação foi apresentada apenas em relação à equação de regressão escolhida para a espécie.

\section{RESULTADOS E DISCUSSÃO}

As folhas de $E$. heterophylla apresentaram $C$ médio de $4,09 \mathrm{~cm}, L$ média de $2,72 \mathrm{~cm}$ e $A f$ média de $7,75 \mathrm{~cm}^{2}$ (Tabela 1), estando $58,5 \%$ da área foliar relacionados com folhas de 6,01 a $9,00 \mathrm{~cm}^{2}$ (Tabela 2). Em comparação a outras plantas daninhas de "folhas largas" já estudadas, a espécie apresentou menor área foliar média que Cissampelos glaberrima (Bianco et al., 2002), Leonotis nepetaefolia (Bianco et al., 2007b) e Typha latifolia (Bianco et al., 2003) e maior que Tridax procumbens (Bianco et al., 2004). Portanto, em relação às espécies supracitadas, as folhas de E. heterophylla

Tabela 1 - Valores máximos, mínimos e médios do comprimento ao longo da nervura central, largura e área foliar de 200 limbos de Euphorbia heterophylla. FCAV/UNESP, Jaboticabal-SP, 2007

\begin{tabular}{|l|c|c|c|}
\hline \multicolumn{1}{|c|}{ Parâmetro } & Maior valor & Menor valor & Média \\
\hline Comprimento $(\mathrm{cm})$ & 6,80 & 2,10 & 4,09 \\
\hline Largura máxima $(\mathrm{cm})$ & 5,80 & 1,60 & 2,72 \\
\hline Área foliar $\left(\mathrm{cm}^{2}\right)$ & 16,94 & 3,36 & 7,75 \\
\hline
\end{tabular}

apresentam área com capacidade de absorção luminosa maior apenas que $T$. procumbens.

Os resultados de regressão efetuados, relacionando $A f \operatorname{com} C, L$ e $C^{*} L$ (Tabela 3 ), indicam que todas as equações apresentadas permitiram obter estimativas satisfatórias da área foliar de $E$. heterophylla, com coeficientes de determinação acima de 0,80 , com exceção da equação linear e da geométrica relacionando $A f$ somente com $C$, cujos coeficientes de determinação estão em torno de 0,50 . As equações que representam $C * L$ passando ou não pela origem não mostraram diferenças significativas quando comparadas entre si, sendo o esperado, visto que a retirada de uma constante não afeta o comportamento dos dados (Neter \& Wasserman, 1974).

Os maiores valores do coeficiente de determinação e os menores valores da soma de quadrados do resíduo foram observados para as regressões lineares entre a $A f$ e $C^{*} L$, indicando serem as equações que permitem obter

Tabela 2 - Distribuição percentual da área foliar real (Af) de 200 limbos foliares de Euphorbia heterophylla, em relação a diferentes faixas de tamanho. FCAV/UNESP, JaboticabalSP, 2007

\begin{tabular}{|c|c|}
\hline $\mathrm{Af}\left(\mathrm{cm}^{2}\right)$ & $(\%)$ \\
\hline$<3,50$ & 0,5 \\
\hline $3,51-6,00$ & 17,0 \\
\hline $6,01-9,00$ & 58,5 \\
\hline $9,01-12,00$ & 20,0 \\
\hline $12,01-15,00$ & 3,5 \\
\hline$>15,01$ & 0,5 \\
\hline
\end{tabular}

Tabela 3 - Equações de regressão estimadas, coeficientes de determinação ( $\left.\mathrm{R}^{2}\right)$, graus de liberdade (GL) e somas de quadrados de desvios da regressão (SQR) da área foliar, em função das medidas lineares do limbo foliar de Euphorbia heterophylla. FCAV/ UNESP, Jaboticabal-SP, 2007

\begin{tabular}{|l|c|c|c|c|c|}
\hline $\mathrm{x}^{(1)}$ & Equação & $\mathrm{R}^{2}$ & $\mathrm{GL}$ & $\begin{array}{c}\text { SQR } \\
\text { (escala original) }\end{array}$ & $\begin{array}{c}\text { Equação Estimada } \\
\text { (Af') }\end{array}$ \\
\hline $\mathrm{C}$ & Linear & 0,5229 & 198 & 693,2974 & $2,1347+1,3819 \mathrm{C}$ \\
\hline $\mathrm{L}$ & Linear & 0,8255 & 198 & 304,0041 & $-0,7554+3,1147 \mathrm{~L}$ \\
\hline $\mathrm{CL}$ & Linear & 0,9184 & 198 & 149,3060 & $1,3005+0,57610 * \mathrm{CL}$ \\
\hline $\mathrm{CL}_{(0,0)}$ & Linear & 0,9184 & 199 & 178,8933 & $0,6816^{*} \mathrm{CL}$ \\
\hline $\mathrm{C}$ & Geométrica & 0,4937 & 198 & 712,5272 & $2,9545^{*} \mathrm{C}^{1,6705}$ \\
\hline $\mathrm{L}$ & Geométrica & 0,8518 & 198 & 327,9176 & $2,2296 * \mathrm{~L}^{1,1963}$ \\
\hline $\mathrm{C}$ & Exponencial & 0,8518 & 198 & 687,4462 & $3,7177 * 1,1869 \mathrm{C}$ \\
\hline $\mathrm{L}$ & Exponencial & 0,8130 & 198 & 583,6007 & $2,5699 * 1,4772 \mathrm{~L}$ \\
\hline
\end{tabular}

${ }_{1 /}$ medidas lineares: comprimento (C) e largura (L) do limbo foliar. 
estimativas mais acuradas da área foliar da planta daninha em estudo. Nota-se que essas equações apresentaram estimativas do coeficiente de determinação de 0,9184 , sugerindo que $91,84 \%$ das variações totais observadas podem ser explicadas pela regressão linear. A equação linear simples, com a reta passando pela origem, é a mais recomendada, pois não altera expressivamente a soma de quadrados do resíduo (Tabela 3) e apresenta análise de resíduos satisfatória (Figura 1), além de ser a de mais fácil utilização do ponto de vista prático, sendo, portanto, a equação escolhida.

Apesar da dispersão de alguns dados na análise de resíduos (Figura 1a), observa-se tendência geral de normalidade na sua distribuição (Figura 1b), confirmada através da não
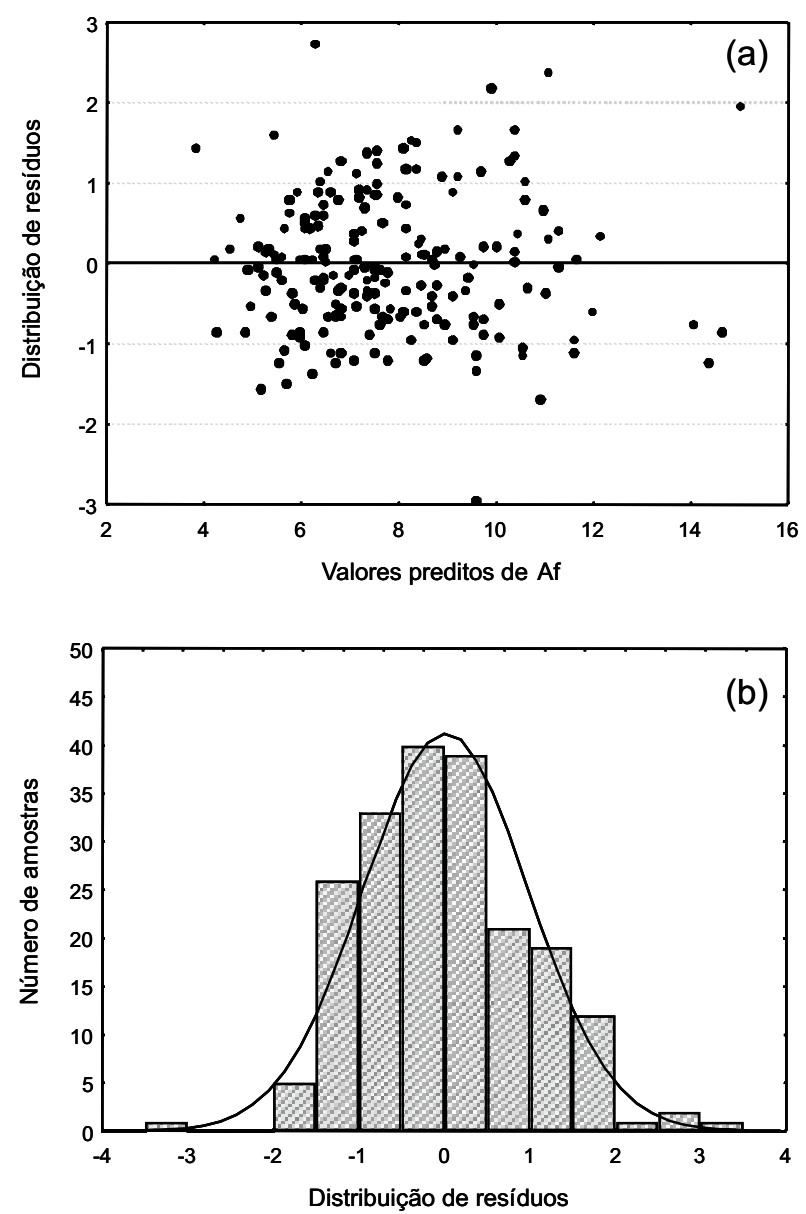

Figura 1 - Análise de resíduos referente à equação de regressão linear simples, de coeficiente linear igual a 0 , caracterizada pela comparação dos valores de área foliar e resíduos (a) e pelo histograma de resíduos (b). FCAV/UNESP, JaboticabalSP, 2007 significância $(p>0,05)$ do teste de normalidade de Shapiro-Wilk, para ambas as espécies. Aceita-se isso para o parâmetro de crescimento estudado, considerando os limites ecológicos extremos no habitat das plantas daninhas, as quais vivem em agroecossistemas com os mais variados estresses e distúrbios ambientais, o que altera o crescimento da planta e aumenta a variabilidade dos dados observados no campo (Bianco et al., 2008a, b).

A análise de correlação de Spearman foi significativa $(p<0,05)$, confirmando a correlação entre os valores reais e os estimados pela equação escolhida; o valor do coeficiente de correlação foi de 0,925 .

Portanto, as análises estatísticas usadas para validar o modelo matemático escolhido foram satisfatórias, considerando o parâmetro biológico estudado; assim, a estimativa da área foliar de $E$. heterophylla pode ser feita por meio da equação linear passando pela origem (Figura 2).

Os resultados encontrados neste trabalho permitem concluir que todas as equações obtidas podem ser empregadas para estimar a área foliar de E. heterophylla, porém, do ponto de vista prático, recomenda-se utilizar a equação $A f^{\prime}=0,6816^{*}\left(C^{*} L\right)$. A determinação dessa equação é ferramenta importante na análise de crescimento de $E$. heterophylla, uma vez que

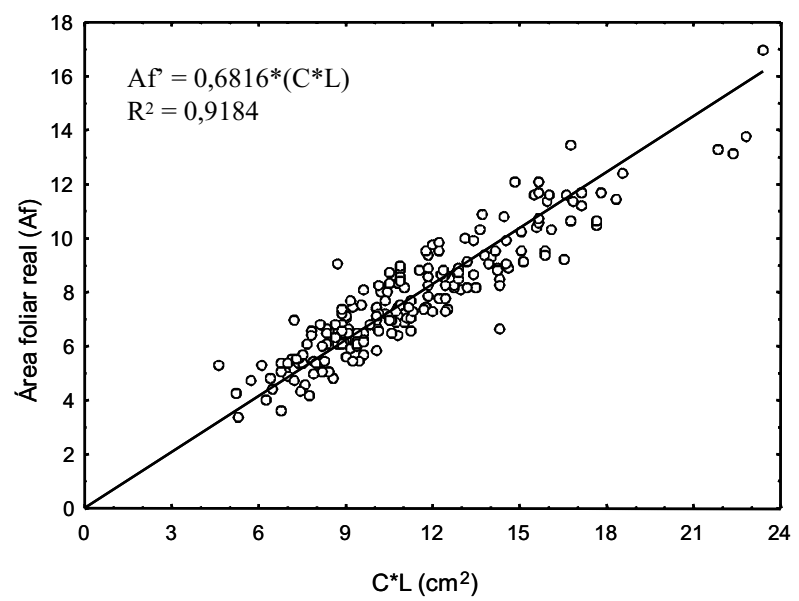

Figura 2 - Representação gráfica da área foliar de Euphorbia heterophylla e da equação de regressão indicada para estimativa da área foliar da planta daninha, em função do produto do comprimento $(\mathrm{C})$ pela largura $(\mathrm{L})$ máxima do limbo foliar. FCAV/UNESP, Jaboticabal-SP, 2007. 
o cálculo da área foliar é imprescindivel para a realização desse estudo.

\section{LITERATURA CITADA}

BIANCO, S.; PITELLI, R.A.; BIANCO, M. S. Estimativa da área foliar de Brachiaria plantaginea usando dimensões lineares do limbo foliar. Planta Daninha, v. 23, n. 4, p. 597-601, 2005.

BIANCO, S.; PITELLI, R. A.; CARVALHO, L. B. Estimativa da área foliar de Cissampelos glaberrima L. usando dimensões lineares do limbo foliar. Planta Daninha, v. 20, n. 3, p. 353-356, 2002.

BIANCO, S. et al. Estimativa da área foliar de Ipomoea hederifolia e Ipomoea nil Roth. usando dimensões lineares do limbo foliar. Planta Daninha, v. 25, n. 2, p. 325-329, 2007a.

BIANCO, S.; BIANCO, M. S.; CARVALHO, L. B. Estimativa da área foliar de Leonotis nepetifolia (L.) W. T. Aiton usando dimensões lineares do limbo foliar. Ci. Cult., v. 2 , n. 1 , p. $27-31,2007 \mathrm{~b}$.

BIANCO, S. et al. Estimativa da área foliar de Ageratum conyzoides usando dimensões lineares do limbo foliar. Acta Sci. Agron., v. 30, n. 4, p. 519-523, 2008 a.

BIANCO, S. et al. Estimativa da área foliar de Sida cordifolia e Sida rhombifolia usando dimensões lineares do limbo foliar. Planta Daninha, v. 26, n. 4, p. 807-813, $2008 b$.
BIANCO, S.; PITELLI, R. A.; CARVALHO, L. B. Estimativa da área foliar de Tridax procumbens usando dimensões lineares do limbo foliar. Planta Daninha, v. 22, n. 2, p. 247-250, 2004

BIANCO, S.; PITELLI, R. A.; PITELLI, A. M. C. M. Estimativa da área foliar de Typha latifolia usando dimensões lineares do limbo foliar. Planta Daninha, v. 21, n. 2, p. $257-261,2003$.

KISSMANN, K. G.; GROTH, D. Plantas infestantes e nocivas. 2.ed. São Paulo: BASF, 1999. Tomo II. 978 p.

MEAD, R.; CURNOW, R. N. Statistical methods in agriculture and experimental biology. New York: Chapman and Hall, 1983. 335 p.

NETER, J; WASSERMAN, W. Applied models. Regressions, analysis of variance and experimental designs. Illinois: Rechard D. Irwin, 1974. $842 \mathrm{p}$.

PEDRO JÚNIOR, M. J.; RIBEIRO, I. J. A.; MARTINS, F. P. Determinação da área foliar em videira cultivar Niagara Rosada. Bragantia, v. 45, n. 1, p. 199-204, 1986.

PERESSIN, V. A.; PITELLI, R. A.; PERECIN, D. Métodos para estimativa da área foliar de plantas daninhas. 4. Cassia tora L. Planta Daninha, v. 7, n. 2, p. 48-52, 1984.

QUEIROGA, J. L. et al. Estimativa da área foliar o feijãovagem (Phaseolus vulgaris L.) por meio da largura máxima do folíolo central. Hortic. Bras., v. 21, n. 1, p. 64-68, 2003.

SILVA, N. F. et al. Modelos para estimar a área foliar de abóbora por meio de medidas lineares. R. Ceres, v. 45, n. 259 , p. 287-291, 1998. 